\title{
ERRATUM
}

\section{Enterococcus flavescens sp. nov., a New Species of Enterococci of Clinical Origin}

RAFFAELLO POMPEI, FRANCESCA BERLUTTI, MARIA C. THALLER, ANGELA INGIANNI, GIAMPIERO CORTIS, AND BENEDETTO DAINELLI

\author{
Istituto di Microbiologia, Università di Cagliari, and Istituto di Medicina Legale, via Porcell 4, \\ Cagliari, Istituto di Microbiologia, Università di Roma "La Sapienza," Rome, and \\ Istituto di Medicina Sperimentale, Università di Chieti, Chieti, Italy
}

Volume 42, no. 3, p. 365, abstract, line 5: "CCM 439" should read "CCM 4239."

Page 366, Table 1, lines 1, 2, and 4: "CCM 439 ${ }^{\mathrm{T}}$," "CCM 440," and "CCM 441" should read "CCM 4239"," "CCM 4240," and "CCM 4241," respectively.

Page 368, column 1, lines 9 and 10: “CCM 439”, should read “CCM 4239." 